\title{
Light controlled optical Aharonov-Bohm oscillations in a single quantum ring
}

Heedae Kim, ${ }^{\dagger, \ddagger}$ Seongho Park ${ }^{\boldsymbol{d}}$ Rin Okuyama, ${ }^{\S}$ Kwangseuk Kyhm, ${ }^{*}$ Mikio Eto, ${ }^{\S}$

Robert A. Taylor, ${ }^{*, \ddagger}$ Gilles Nogues,,$"$ Le Si Dang," Marek Potemski, ${ }^{\perp}$ Koochul Je,\# Jongsu Kim, ${ }^{@}$ Jihoon Kyhm, ${ }^{\triangle}$ and Jindong Song ${ }^{\triangle}$

$\dagger$ School of Physics, Northeast Normal University, Changchun 130024, China

$\ddagger$ Clarendon Laboratory, Department of Physics, University of Oxford, Oxford, OX1 3PU,

$$
U . K
$$

\Department of Opto-mechatronics, Cogno-mechatronics, Physics Education, Pusan Nat'l University, Busan 609-735, South Korea

§Faculty of Science and Technology, Keio University, Yokohama 223-8522, Japan

||Department of NANOscience, Institut Néel, CNRS, rue des Martyrs 38054, Grenoble,

France

$\perp$ Laboratoire National des Champs Magnetiques Intenses, CNRS-UJF-UPS-INSA, F-38042, Grenoble, France

\#Institute of Physics, Faculty of Natural Science and Mathematics, Sts Cyril and Methodius University, 1000 Skopje, Macedonia

@Department of Physics, Yeungnam University, Gyeonsan 712-749, South Korea $\triangle$ Center for Opto-Electronic Convergence Systems, KIST, Seoul, 136-791, South Korea

E-mail: kskyhm@pusan.ac.kr; robert.taylor@physics.ox.ac.uk

\footnotetext{
${ }^{a}$ equal contribution with first author
}

\section{Abstract}


We found that optical Aharonov-Bohm oscillations in a single GaAs/GaAlAs quantum ring can be controlled by excitation intensity. With a weak excitation intensity of $1.2 \mathrm{kWcm}^{-2}$, the optical Aharonov-Bohm oscillation period of biexcitons was observed to be half that of excitons in accordance with the period expected for a two-exciton Wigner molecule. When the excitation intensity is increased by an order of magnitude $\left(12 \mathrm{kWcm}^{-2}\right)$, a gradual deviation of the Wigner molecule condition occurs with the decreased oscillation periods and diamagnetic coefficients of both excitons and biexcitons along with a spectral shift. These results suggest that the effective orbit radii and rim widths of electrons and holes in a single quantum ring can be modified by light intensity via photo-excited carriers, which are possibly trapped at interface defects resulting in a local electric field.

The Aharonov-Bohm (AB) effect has been investigated mostly by electrical experiments in mesoscopic ring structures, where oscillations of the conductance and persistent current with external magnetic field $(B)$ were observed at extremely low temperature $(<100 \mathrm{mK})$ due to the fragile quantum coherence in sub-micron sized systems. ${ }^{1-6}$ Recently, the AB effect became accessible to optical experiments by using type-II quantum dots (QDs) and quantum rings (QRs), where quantum coherence on a nanometer scale $(\sim 50 \mathrm{~nm})$ is observed at temperatures up to three orders of magnitude higher $(<100 \mathrm{~K}) \cdot{ }^{7-12}$

While either an individual electron or hole rotates in the shell of a type-II QD, both electrons and holes orbit around the circumference of a QR. When a strong Coulomb interaction is present between the electron and the hole in a $\mathrm{QR}$, the exciton $(\mathrm{X})$ can be approximated by a single particle. However, charge neutral Xs require different orbital radii for the electron and the hole $\left(R_{\mathrm{e}} \neq R_{\mathrm{h}}\right)$ in order to give rise to the $\mathrm{AB}$ effect. ${ }^{6}$ In this case, a difference between the magnetic fluxes threading their orbits $\left(\Phi=B \pi\left|R_{\mathrm{e}}^{2}-R_{\mathrm{h}}^{2}\right|\right)$ determines the phase of a radially polarized X $(\phi=e \Phi / \hbar)$. Therefore, oscillations in the X energy can be observed optically as $B$ increases. When a one-dimensional (1D) model is assumed for a QR structure, the X AB oscillation period $\Delta B_{\mathrm{X}}=(h / e) /\left(\pi\left|R_{\mathrm{e}}^{2}-R_{\mathrm{h}}^{2}\right|\right)$ can be estimated.

On the other hand, when $N$ electrons are strongly correlated in a ring structure, they can 
behave as a composite rigid body, called a Wigner molecule (WM). ${ }^{13-16}$ In this case, the AB oscillation period of an $N$-electron WM with applied $B$ becomes fractional $\left(\Delta B_{N \mathrm{e}}=\Delta B_{\mathrm{e}} / N\right)$ when compared to that of a single electron $\Delta B_{\mathrm{e}}=(h / e) /\left(\pi R_{\mathrm{e}}^{2}\right),{ }^{17-20}$ and the presence of a two-electron WM has been claimed in a large quantum dot ${ }^{14-16}$ and a clean carbon nanotube $^{13}$ by electrical measurements. In an optical analogy, biexcitons (XXs) in a QR can be a two-X WM. ${ }^{9}$ If a pair of Xs located at opposite positions in a QR are strongly correlated like a rigid dumbbell, the $\mathrm{AB}$ oscillation period of the $\mathrm{XX}$ WM $\left(\Delta B_{\mathrm{XX}}\right)$ is expected to be half that of the $\mathrm{X}\left(\Delta B_{\mathrm{X}}\right)$, i.e. $\Delta B_{\mathrm{X}} / \Delta B_{\mathrm{XX}}=2$.

Although the orbits of electrons and holes in a QR are often simplified into 1 dimensional loops, the rim width is considerable in comparison to the QR radius. In this case, the wavefunctions and the Coulomb interaction of electrons and holes in a finite rim width need to be considered to predict optical AB oscillations of X and XX, and the two-X WM criterion is also known to be vulnerable to the Coulomb interaction. Nevertheless, this subtle range has barely been investigated experimentally so far. In addition, the presence of a local electric field $\left(E_{\mathrm{Loc}}\right)$ is quite likely at the interface between a QR and the capping barrier material. Suppose that carriers excited in a capping barrier affect $E_{\text {Loc }}$ at the interface, the wavefunction modification of a QR might be susceptible to optical excitation intensity changes. In this work, we have found that optical AB oscillations of both Xs and XXs in a single GaAs/AlGaAs QR, can be controlled by the optical excitation intensity, whereby the two-X WM criterion is scrutinised in terms of $\Delta B_{\mathrm{X}} / \Delta B_{\mathrm{XX}}$.

For the growth of a GaAs QR on AlGaAs substrates, gallium molecular beams are supplied initially without arsenic ambient, leading to the formation of droplet-like clusters of gallium atoms on the substrate. ${ }^{21,22}$ As an arsenide flux is supplied in the chamber, the gallium droplet becomes crystallised into GaAs ring structures, where the morphology is governed by the interplay between the migration of surface adatoms and their crystallisation. In Fig. 1(a) and (b), the rim height of an uncapped GaAs QR grown by the droplet epitaxy ${ }^{21,22}$ is anisotropic, where a difference between the maximum and minimum rim height along [1̄ㅣ] 

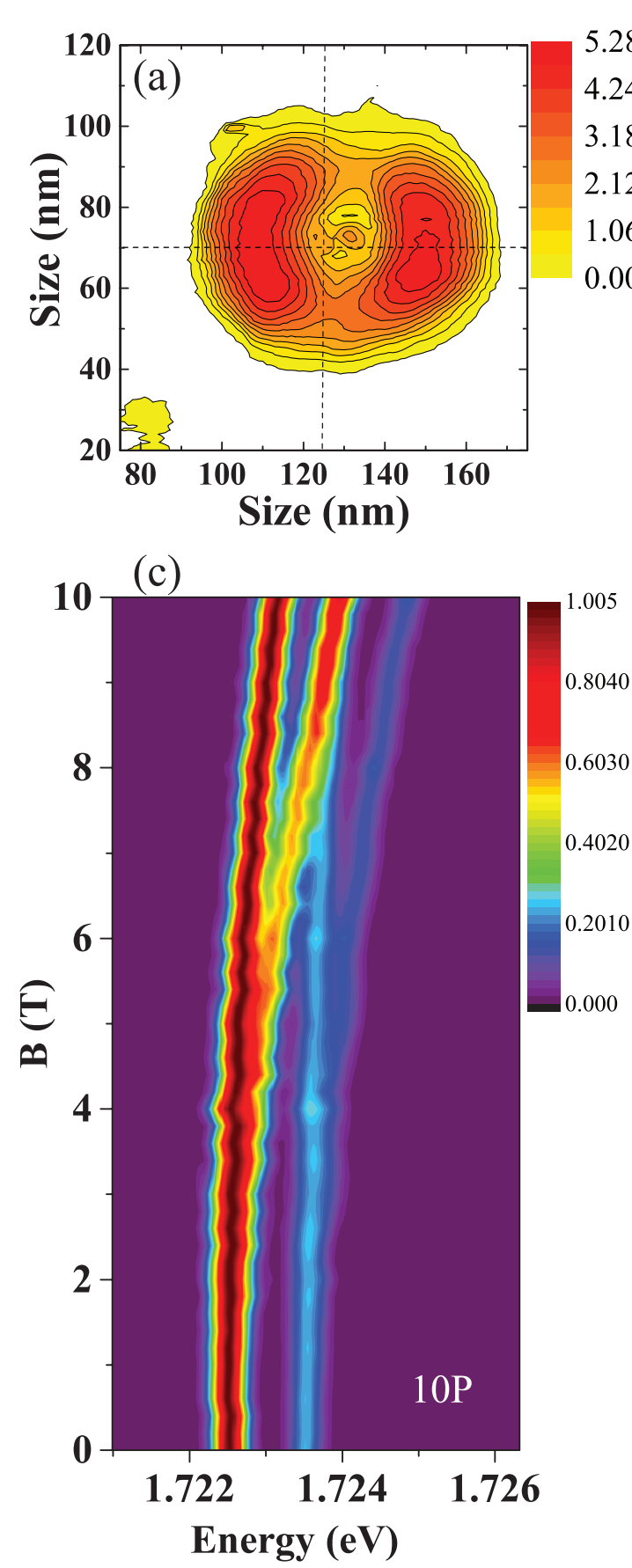

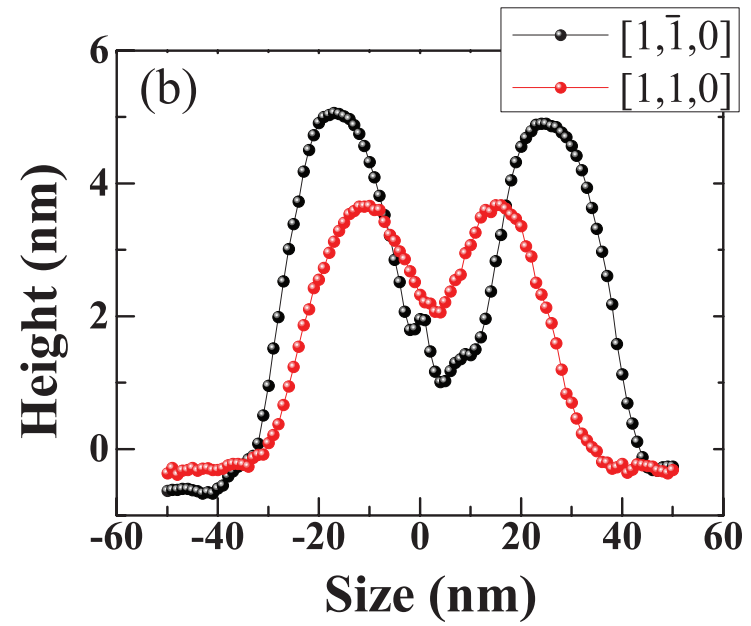

(d)

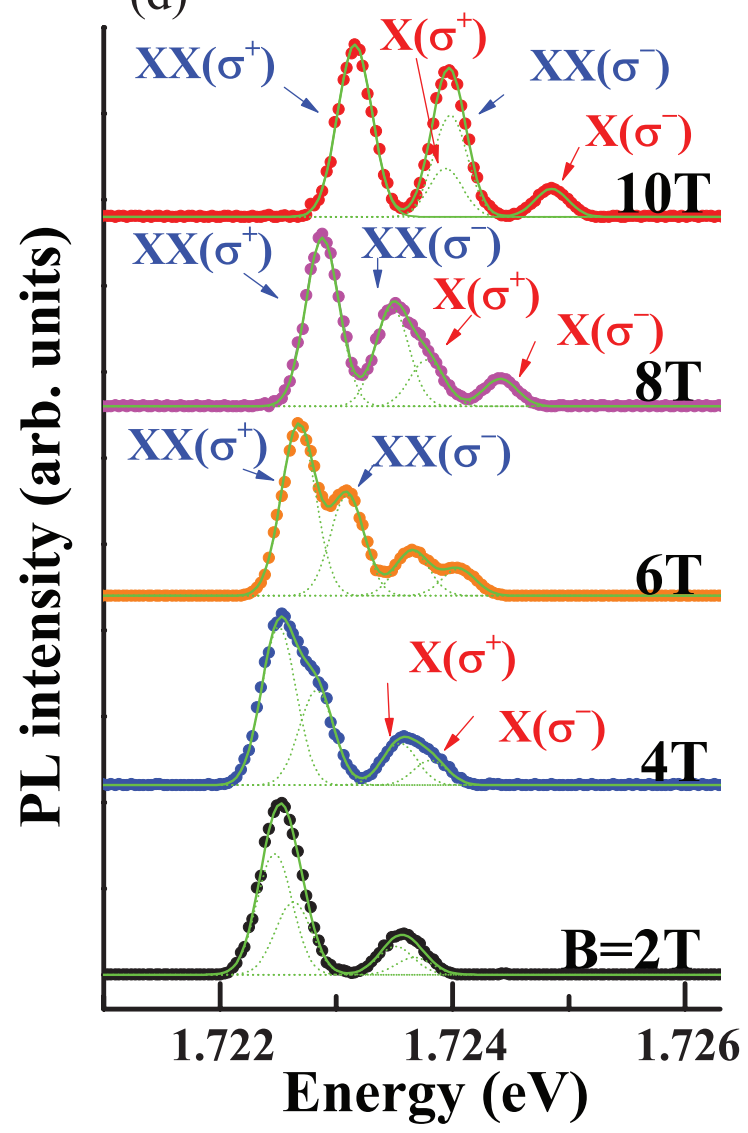

Figure 1: AFM images of a single GaAs QR in a top (a) and side (b) view. (c) With an excitation intensity of $12 \mathrm{kWcm}^{-2}(10 P)$, the micro-PL spectra of a single GaAs/AlGaAs QR were observed as a function of $B$, where the Zeeman doublet spectra of X and XX were fitted by Gaussian functions (d). 
and [110] is $\sim 2 \mathrm{~nm}$, and the lateral size is also elongated along [110]. In this volcano-like structure, the wavefunction is likely to be localised in a crescent-like shape unless sufficient magnetic field $B$ is applied, ${ }^{23-26}$ and the two separate localised states become merged beyond a characteristic magnetic field $\left(B_{\mathrm{c}}\right)$. As a result, AB oscillations of a QR begin when $B \geq B_{\mathrm{c}}{ }^{5}$

As the excitation intensity $\left(I_{\mathrm{ex}}\right)$ increases, an additional PL peak becomes significant at $\sim 1$ meV below the X PL spectrum, which has been attributed to XX by the quadratic $I_{\mathrm{ex}}$ dependence of the integrated PL intensity $\left(\sim I_{\mathrm{ex}}^{2}\right)$ and the short PL decay time $\left(\tau_{\mathrm{XX}} \sim 370 \mathrm{ps}\right)$ compared to that of $\mathrm{X}\left(\tau_{\mathrm{X}} \sim 820 \mathrm{ps}\right)$. Although charged excitons are rarely seen at either $\sim 4 \mathrm{meV}$ below and $\sim 2 \mathrm{meV}$ above the X PL spectrum, a charged exciton-free QR was carefully selected. As shown in Fig. 1(c), the micro-PL spectrum of a single QR was measured at $4 \mathrm{~K}$ for increasing $B$ by using a resistive DC magnet with $52 \mathrm{~mm}$ bore diameter, where excitation by a continuous-wave $\mathrm{Ar}^{+}$laser $(488 \mathrm{~nm})$ was introduced to the position-controlled sample through a multi-mode fiber, and the PL was collected through a single-mode fiber. The wiggling of the energy for $B$ in Fig. 1(c) became clarified by plotting the PL peak emission energies of the Zeeman doublet $\left(E_{\mathrm{PL}}\left(\sigma^{+}\right)\right.$and $\left.E_{\mathrm{PL}}\left(\sigma^{-}\right)\right)$, which were obtained by Gaussian fitting with an accuracy of $\sim 2.5 \mu \mathrm{eV}$ (Fig. 1(d)). As the Zeeman doublets of $\mathrm{X}$ and XX become resolvable for $B>2 \mathrm{~T}$ (Fig.1 (d)), a ring shape wavefunction is possibly formed from separate crescent-like ones possibly near $B_{\mathrm{c}} \sim 2 \mathrm{~T}$.

Recently, we have studied a strongly localised exciton state in a GaAs $Q R,{ }^{26}$ and the lateral wavefunction shape was known to be crescent-like. For a micro-PL spectrum near $1.812 \mathrm{eV}$ of the strongly localised state, we found both the excitonic $g$-factor $(\sim-0.2)$ and diamagnetic coefficient $\left(\gamma_{\mathrm{X}} \sim 1.3 \mu \mathrm{eVT}^{-2}\right)$ were very small. Therefore, the Zeeman splitting was not significant unless strong magnetic field $(B>4 \mathrm{~T})$ is applied. On the other hand, when a relatively low energy micro-PL spectrum $(\sim 1.732 \mathrm{eV})$ was investigated in a GaAs $\mathrm{QR},{ }^{9}$ we found the Zeeman doublet spectra of X and XX become separable near $2 \mathrm{~T}$, and the excitonic $g$-factor $(\sim-1.3)$ and diamagnetic coefficient $\left(\gamma_{\mathrm{X}} \sim 1.3 \mu \mathrm{eVT}^{-2}\right)$ are enhanced 

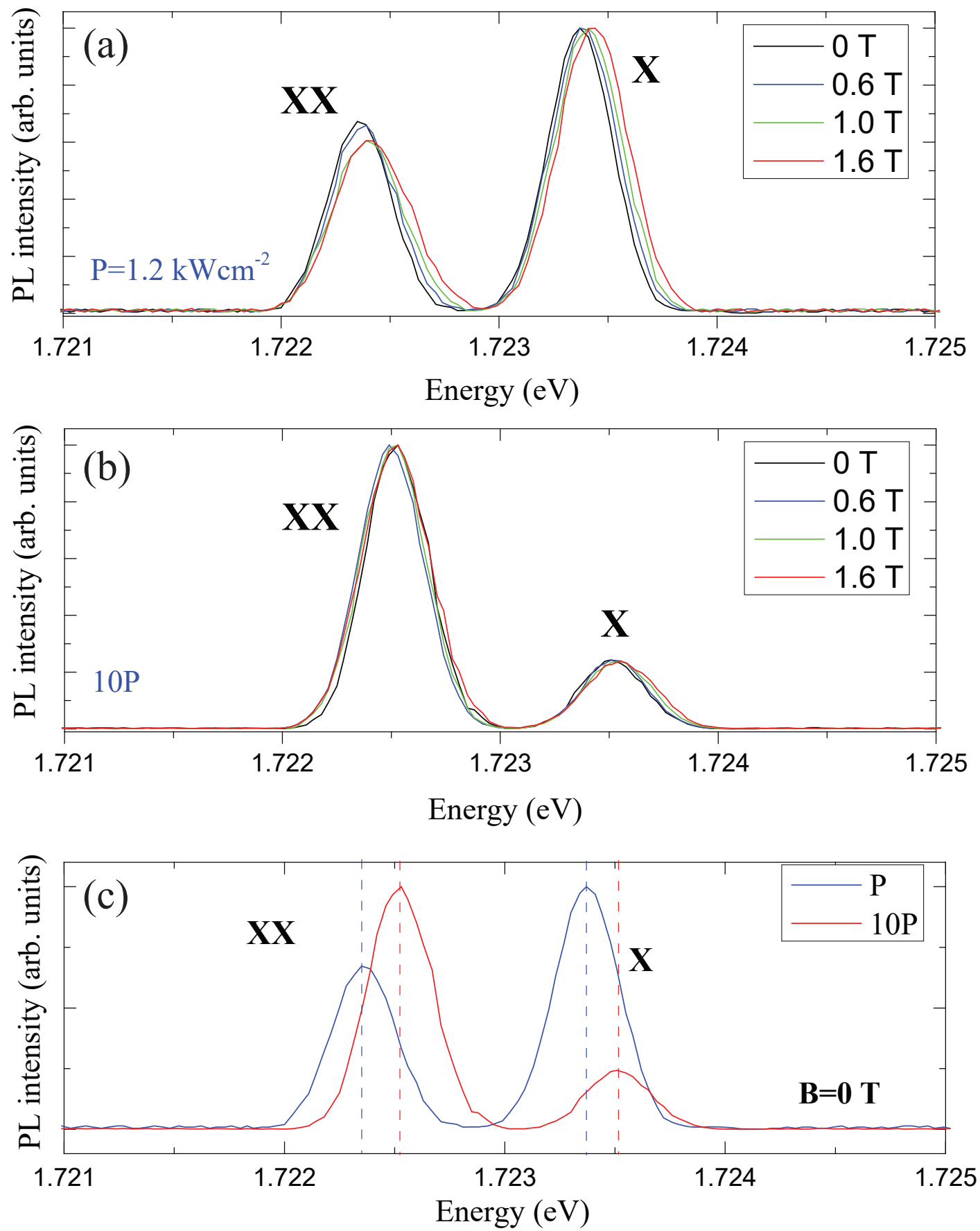

Figure 2: PL spectra of $\mathrm{X}$ and $\mathrm{XX}$ are measured under weak $\left(P=1.2 \mathrm{kWcm}^{-2}\right)(\mathrm{a})$ and strong excitation $(10 P)$ (b) for increasing $B$ up to $1.6 \mathrm{~T}$, where the Zeeman doublets look inseparable. (c) When excitation is increased from $P$ to $10 P$ in the absence of $B$, both $\mathrm{X}$ and XX show a blueshift in PL spectrum. 
by an order of magnitude compared to those of the strongly localised state. Therefore, one may suggest that $B_{\mathrm{c}}$ seems to depend on the degree of localisation and the QR anisotropy. While strongly localised states in a volcano-like $\mathrm{QR}$ require a large $B_{\mathrm{c}}$ to give rise to $\mathrm{AB}$ oscillations, a small $B_{\mathrm{c}}$ is expected for the weakly localised states. As shown in Fig. 2(a) and (b), the Zeeman doublet spectra of X and XX are not as separated as energy shift of photoluminescence spectrum unless sufficient magnetic field $(B>2 \mathrm{~T})$ is applied. Therefore, those PL spectra can be attributed to localised states in a QR. In Fig. 2(c), both X and XX show a blueshift for increasing excitation in the absence of $B$. As carriers are excited non-resonantly in the barrier, the blushift is possibly associated with excitation-induced local field $E_{\text {Loc }}$.

$B$-dependence of the average energy $\left[E_{\mathrm{PL}}\left(\sigma^{+}\right)+E_{\mathrm{PL}}\left(\sigma^{-}\right)\right] / 2$ of X (Fig. 3(a),(b), and (c)) and XX (Fig. 3(d), (e), and (f)) were plotted for three different excitations of $P$, $5 P$, and $10 P$, respectively. All show clear AB oscillations along with a diamagnetic energy increase, where the diamagnetic coefficients $(\gamma)$ were obtained by a quadratic fitting $\sim \gamma B^{2}$. In Fig. 3(g), $\left[E_{\mathrm{PL}}\left(\sigma^{+}\right)-E_{\mathrm{PL}}\left(\sigma^{-}\right)\right] / 2$ of $\mathrm{X}$ and $\mathrm{XX}$ were also plotted, and a linear fitting function of $g \mu_{\mathrm{B}} B$ enables to estimate the $g$-factors of $\mathrm{X}\left(g_{\mathrm{X}} \sim-1.61 \pm 0.05\right)$ and XX $\left(g_{\mathrm{XX}} \sim-1.31 \pm 0.08\right)$. A subtle deviation from the linear dependence may be explained by $B$-dependence of $g$-factor, and this is possibly associated with a wavefunction modification in a QR structure. On the other hand, the diamagnetic coefficients of both $\mathrm{X}\left(\gamma_{\mathrm{X}}\right)$ and XX $\left(\gamma_{\mathrm{xx}}\right)$ decrease by $20 \%($ Fig. $3(\mathrm{~h}))$ as the excitation intensity increases from $1.2 \mathrm{kWcm}^{-2}$ $(P)$ to $12 \mathrm{kWcm}^{-2}(10 P)$. Because a diamagnetic coefficient is proportional to its lateral wavefunction area, the decrease of $\gamma_{\mathrm{X}}$ and $\gamma_{\mathrm{XX}}$ implies a shrinkage of the wavefunction rim width for excitation. Additionally, both $\mathrm{X}$ and $\mathrm{XX}$ show a blueshift of $\sim 0.2 \mathrm{meV}$ at $B=2 \mathrm{~T}$ for an order of magnitude increase of excitation intensity from $P$ to $10 P$. Therefore, excited carriers seem to change the potential energy possibly via $E_{\text {Loc }}$, resulting in a modification of the ring orbit wavefunctions.

As a prerequisite condition for an optical AB effect, a built-in electric field in a $Q R$ is 

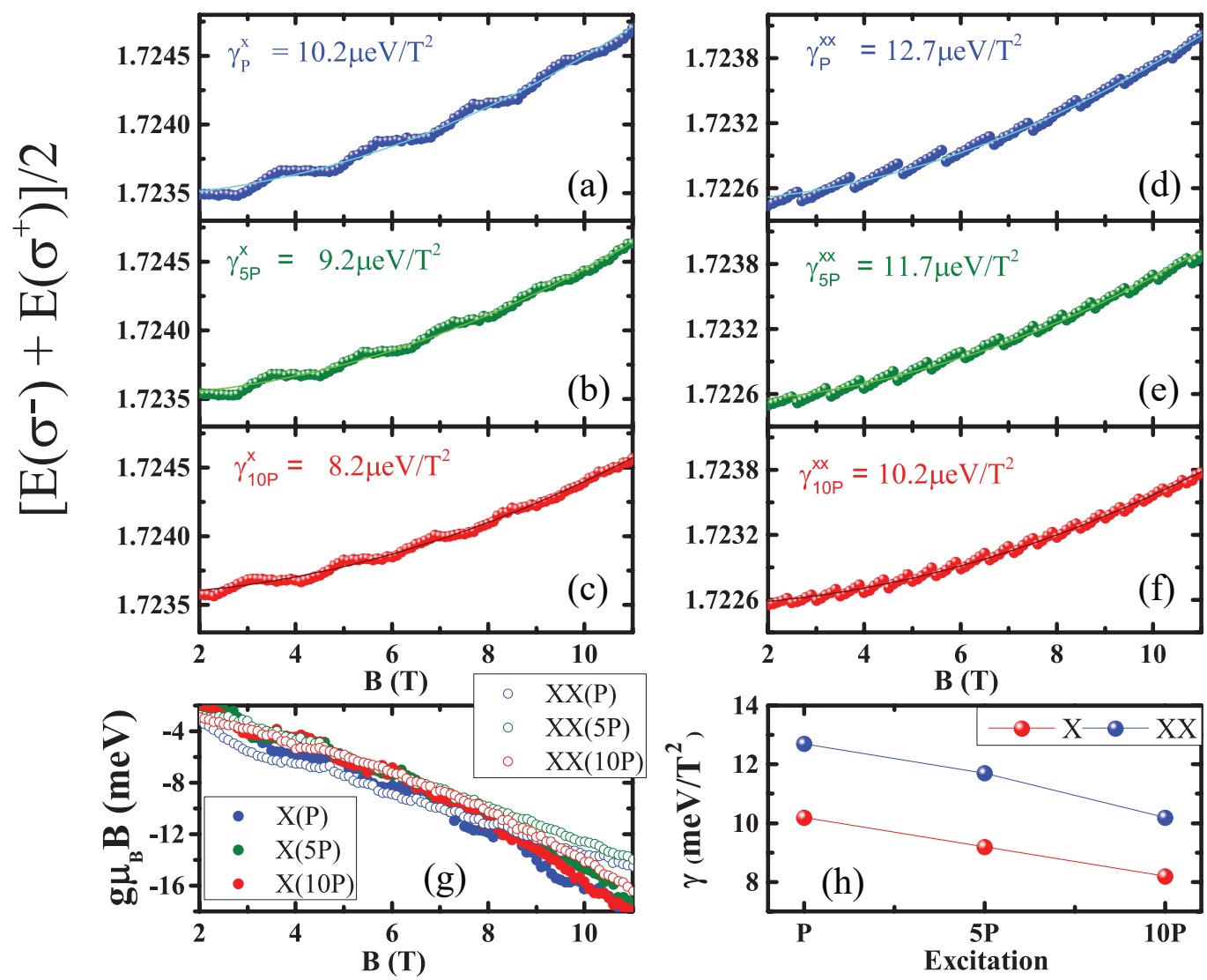

Figure 3: (a) $\left[E_{\mathrm{PL}}\left(\sigma^{+}\right)+E_{\mathrm{PL}}\left(\sigma^{-}\right)\right] / 2$ of $\mathrm{X}(\mathrm{a}, \mathrm{b}, \mathrm{c})$ and $\mathrm{XX}(\mathrm{d}, \mathrm{e}, \mathrm{f})$ with $B$ are plotted to obtain the diamagnetic coefficient $\gamma$ for $P, 5 P$, and $10 P$ excitation, respectively. (g) $\left[E_{\mathrm{PL}}\left(\sigma^{+}\right)-E_{\mathrm{PL}}\left(\sigma^{-}\right)\right]$of X (filled circle) and XX (open circle) are assumed to be $g \mu_{\mathrm{B}} B$ in order to obtain $g$-factor for $P, 5 P$, and $10 P$ excitation, respectively. (h) The diamagnetic coefficients of $\mathrm{X}$ and $\mathrm{XX}$ are decreased for increasing excitation power. 
known to play a crucial role in the charge separation $\left(R_{\mathrm{e}} \neq R_{\mathrm{h}}\right) \cdot{ }^{6}$ Provided that the tunneling towards the direction away from the ring centre is dominated by light electrons, the heavy hole resides near the ring centre. In this case, $R_{\mathrm{e}}>R_{\mathrm{h}}$ (Fig. 4(a)) can be assumed. As strain due to indium is known to induce an internal electric field in InAs and InGaAs QRs, ${ }^{27,28}$ it is advantageous for the charge separation. Although GaAs QRs are strain-free during the gallium droplet formation and crystallisation, a surface surfactant and low substrate temperature are used in order to suppress the migration length of gallium atoms. Therefore, Ga-rich growth conditions and the lack of As-interdiffusion may give rise to defects at the interface between the GaAs QR and AlGaAs barrier such as Ga-antisites and As-vacancies. Consequently, trapped carriers at the defects result in a localised electric field $E_{\mathrm{Loc}}$. Suppose optically excited carriers affect $E_{\mathrm{Loc}}$, a potential energy change can be induced in a QR. This process may give rise to an excitation intensity dependence of the PL energy, diamagnetic coefficient, and $\mathrm{AB}$ oscillation period.

When an electron-hole pair in a QR is considered (Fig. 4(a) and Supporting Information), the total orbital angular momenta $\left(\ell_{\mathrm{X}}=\ell_{\mathrm{e}}+\ell_{\mathrm{h}}\right)$ is given by the sum of the individual orbital angular momentum of an electron and a hole, and the $\mathrm{X}$ orbit radius $R_{\mathrm{X}}=\left[\left(m_{\mathrm{e}} R_{\mathrm{e}}^{2}+m_{\mathrm{h}} R_{\mathrm{h}}^{2}\right) /\left(m_{\mathrm{e}}+m_{\mathrm{h}}\right)\right]^{1 / 2}$ is defined in terms of the orbit radii $\left(R_{\mathrm{e}, \mathrm{h}}\right)^{?}$ and the effective masses $\left(m_{\mathrm{e}, \mathrm{h}}\right)$ for an electron and a hole. According to the simplified one-dimensional (1D) model, the eigenstates can be given by $\Psi_{\mathrm{X}}^{\ell_{\mathrm{X}}}\left(\theta_{\mathrm{X}}, \phi\right)=\mathrm{e}^{\mathrm{i} \ell_{\mathrm{x}} \theta_{\mathrm{x}}} \psi^{\ell_{\mathrm{x}}}(\phi)$, where two azimuthal angles are defined in the centre-of-mass (CM) coordinate $\left(\theta_{\mathrm{X}}=\frac{m_{\mathrm{e}} R_{\mathrm{e}}^{2} \phi_{\mathrm{e}}+m_{\mathrm{h}} R_{\mathrm{h}}^{2} \phi_{\mathrm{h}}}{m_{\mathrm{e}} R_{\mathrm{e}}^{2}+m_{\mathrm{h}} R_{\mathrm{h}}^{2}}\right)$ and the relative coordinate $\left(\phi=\phi_{\mathrm{e}}-\phi_{\mathrm{h}}\right)$ with the individual azimuthal angles of an electron and a hole $\left(\phi_{\mathrm{e}, \mathrm{h}}\right)$ respectively. The Coulomb interaction in the relative coordinate is given by

$$
V_{\mathrm{eh}}=-\frac{e^{2}}{4 \pi \epsilon}\left[\left(R_{\mathrm{e}}-R_{\mathrm{h}}\right)^{2}+4 R_{\mathrm{e}} R_{\mathrm{h}} \sin ^{2}\left(\frac{\phi_{\mathrm{e}}-\phi_{\mathrm{h}}}{2}\right)\right]^{-1 / 2} .
$$

Provided that the e-h pair is tightly bound with the total mass $m_{\mathrm{X}}=m_{\mathrm{e}}+m_{\mathrm{h}}$, the relative motion can be described by a Gaussian function, $\psi^{\ell_{\mathrm{x}}}(\phi) \propto \exp \left\{-\phi^{2} /\left(2 \xi_{\mathrm{X}}^{2}\right)\right\}$ through the 
(a)
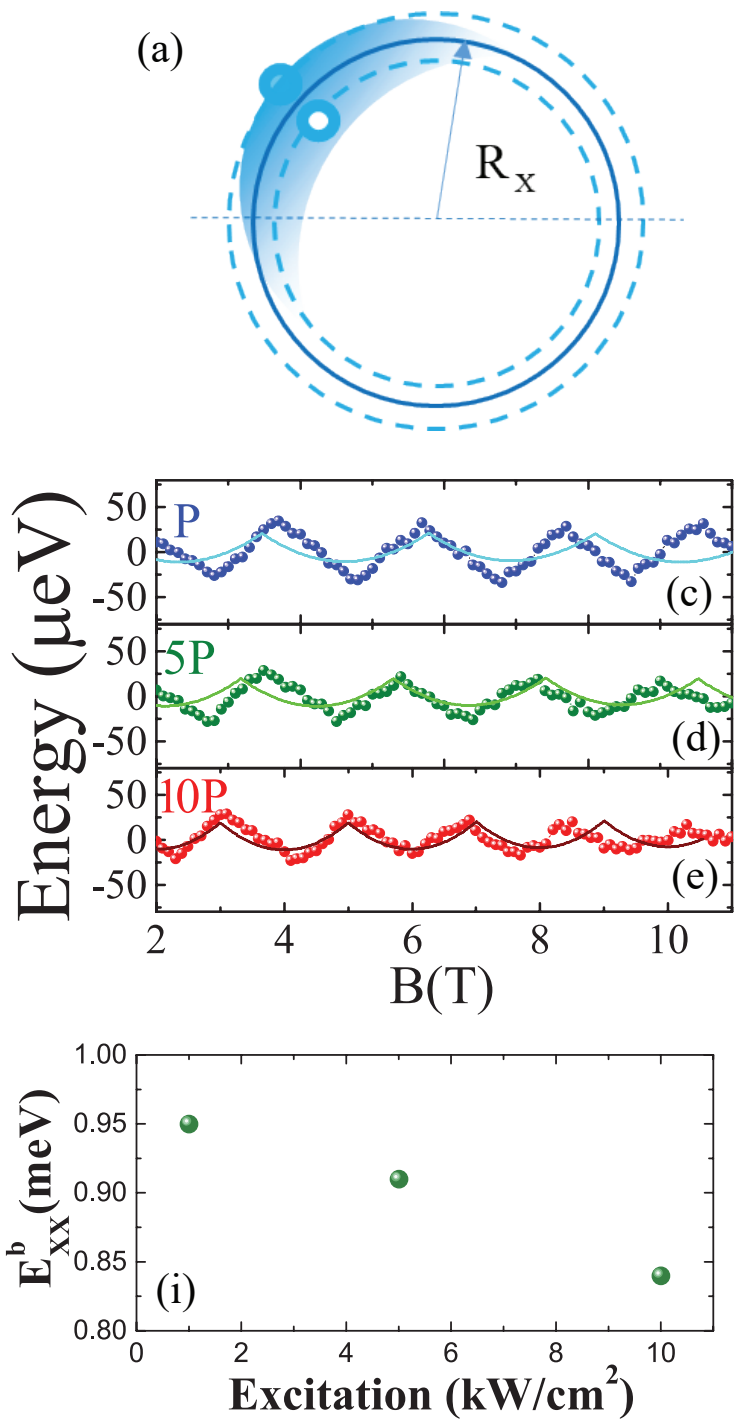

(b)

b)
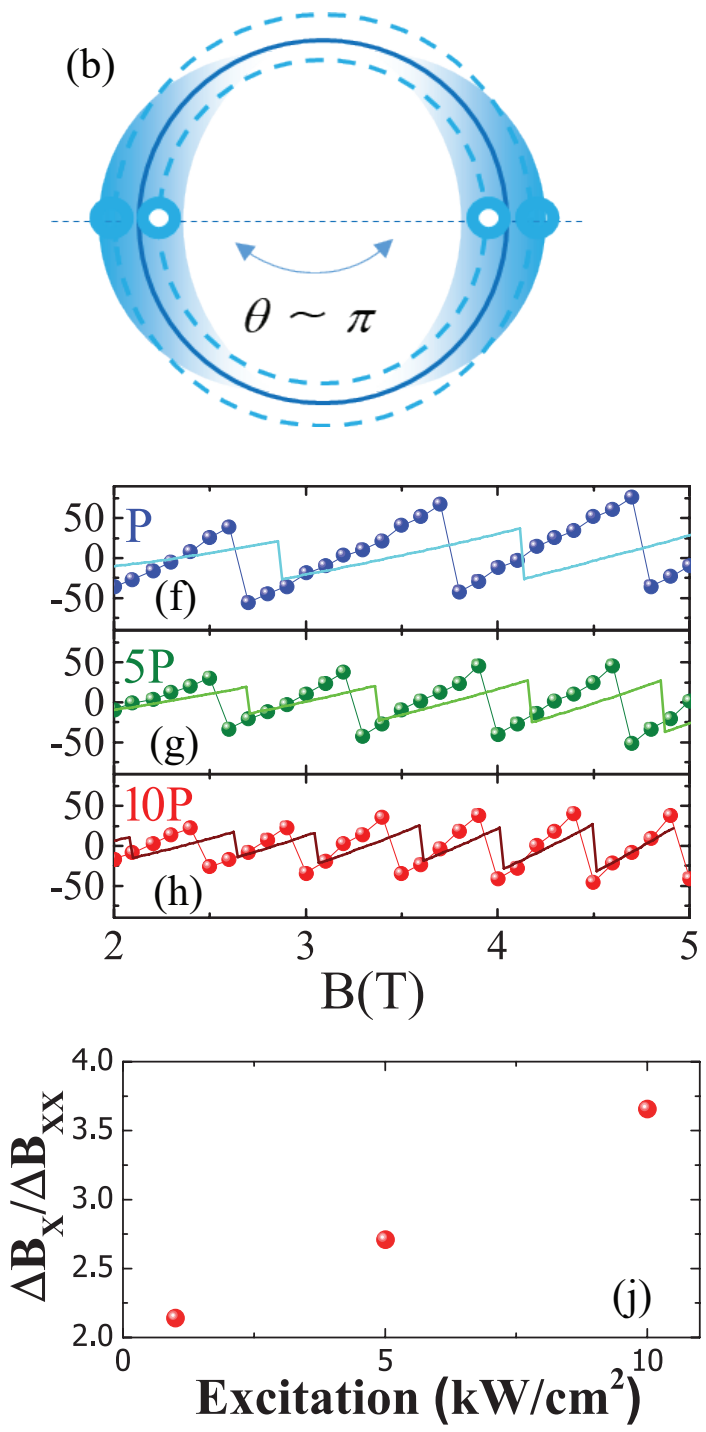

Figure 4: Schematic diagram of X (a) and XX (b) in a QR. Optical AB oscillations of X $(\mathrm{c}, \mathrm{d}, \mathrm{e})$ and XX (f,g,h) were obtained after removing the fitted quadratic functions for an excitation of $P=1.2 \mathrm{kWcm}^{-2}, 5 P$, and $10 P$. Theoretical AB oscillations (solid line) were also compared. The XX binding energy in the absence of $B$ (i) and $\Delta B_{\mathrm{X}} / \Delta B_{\mathrm{XX}}$ (j) vary for increasing excitation. 
second order approximation for $\phi$. If the $\mathrm{X}$ localisation angle is small enough $\left(\xi_{\mathrm{X}}<2 \pi\right)$, the $\mathrm{X}$ energy in the relative coordinate $\left(E_{\mathrm{X}}^{\mathrm{rel}}\right)$ becomes independent of $\ell_{\mathrm{X}}$. Therefore, the electron-hole pair in a QR can be considered as a single particle, and the energy of a rotating $\mathrm{X}$ in a QR under an external $B$ becomes dominated by the energy in the CM coordinate as

$$
E_{\mathrm{X}}^{\ell \mathrm{x}}(B)=\frac{\hbar^{2}}{2 m_{\mathrm{X}} R_{\mathrm{X}}^{2}}\left[\ell_{\mathrm{X}}+\frac{\pi\left(R_{\mathrm{e}}^{2}-R_{\mathrm{h}}^{2}\right) B}{h / e}\right]^{2}+E_{\mathrm{X}}^{\mathrm{rel}}
$$

where $E_{\text {rel }}$ contributes as a $B$-independent constant.

Likewise, a XX in a QR can be considered with a pair of localised Xs (Fig. 4(b) and Supporting Information), where the total XX orbital angular momentum $\left(\ell_{\mathrm{XX}}=\ell_{\mathrm{X}_{1}}+\ell_{\mathrm{X}_{2}}\right)$ is given by the sum of the two individual $\mathrm{X}$ orbital angular momenta $\left(\ell_{\mathrm{X}_{1}}\right.$ and $\left.\ell_{\mathrm{X}_{2}}\right)$. In this case, the dipole-dipole interaction between a pair of $\mathrm{X}$ dipoles can be described as

$$
V_{\mathrm{dd}}(\theta)=\frac{e^{2}\left(R_{\mathrm{e}}-R_{\mathrm{h}}\right)^{2}}{4 \pi \epsilon R_{\mathrm{X}}^{3}} \frac{\left(1+\sin ^{2} \frac{\theta}{2}\right)}{8\left|\sin ^{3} \frac{\theta}{2}\right|}
$$

, which depends on the azimuthal angle difference $\left(\theta=\theta_{1}-\theta_{2}\right)$ of the two Xs. $V_{\mathrm{dd}}(\theta)$ becomes minimised when the two Xs are maximally separated, i.e. $\theta=\pi$. Thus, the XX wavefunction of the relative motion becomes localised near $\theta=\pi$ as $\psi(\theta) \propto \exp \left[-(|\theta|-\pi)^{2} /\left(2 \xi_{\mathrm{XX}}^{2}\right)\right]$, and $\xi_{\mathrm{XX}} \approx\left[R_{\mathrm{X}} /\left(R_{\mathrm{e}}-R_{\mathrm{h}}\right)\right]^{5 / 4} \xi_{\mathrm{X}}$ can be used to characterise the two-X WM. If the quantum fluctuation size is small $\left(\xi_{\mathrm{XX}}<\pi / 2\right)$, the XX energy of the relative motion $\left(E_{\mathrm{XX}}^{\mathrm{rel}}\right)$ can be considered $\ell_{\mathrm{XX}}$-independent. Consequently, the total $\mathrm{XX}$ energy is also dominated by the energy of two Xs in the CM coordinate, and $E_{\mathrm{XX}}^{\mathrm{rel}}$ becomes a $B$-independent constant as

$$
E_{\mathrm{XX}}^{\ell_{\mathrm{XX}}}(B)=\frac{\hbar^{2}}{2\left(2 m_{\mathrm{X}}\right) R_{\mathrm{X}}^{2}}\left[\ell_{\mathrm{XX}}+2 \frac{\pi\left(R_{\mathrm{e}}^{2}-R_{\mathrm{h}}^{2}\right) B}{h / e}\right]^{2}+E_{\mathrm{XX}}^{\mathrm{rel}}
$$

By plotting Eq. (2) for $B$, a series of parabolas are overlapped for various $\ell_{\mathrm{X}}$ states, and the $\mathrm{X}$ orbital angular momentum changes from $\ell_{\mathrm{X}}$ to $\ell_{\mathrm{X}}-1$ on the cusps of $\mathrm{X} \mathrm{AB}$ oscillations to minimise the energy. Therefore, the $\mathrm{X} A \mathrm{AB}$ oscillation period is given by 
$\Delta B_{\mathrm{X}}=(h / e) /\left(\pi\left|R_{\mathrm{e}}^{2}-R_{\mathrm{h}}^{2}\right|\right)$. On the other hand, a saw-like abrupt decrease of the XX energy is seen at the first transition magnetic field where $\ell_{\mathrm{X}}$ changes. This can be explained by the selection rule for the transition between $E_{\mathrm{XX}}^{\ell_{\mathrm{Xx}}}(B)$ and $E_{\mathrm{X}}^{\ell_{\mathrm{X}}}(B)$. For example, considering the XX energy near the transition magnetic field where $\ell_{\mathrm{Xx}}$ changes from 0 to -1 to minimise energy, $E_{\mathrm{X}}^{\ell_{\mathrm{X}}=0}(B)$ still remains less than $E_{\mathrm{X}}^{\ell_{\mathrm{X}}=-1}(B)$ due to the long $\mathrm{AB}$ oscillation period of the minimum $E_{\mathrm{X}}^{\ell_{\mathrm{X}}}(B)$ compared to that of the minimum $E_{\mathrm{XX}}^{\ell_{\mathrm{XX}}}(B)$. Therefore, the selection rule enforces the XX emission transition from $E_{\mathrm{XX}}^{\ell_{\mathrm{XX}}=-1}(B)$ to $E_{\mathrm{X}}^{\ell_{\mathrm{X}}=-1}(B)$, and the abrupt decrease of XX PL energy corresponds to the energy difference between $E_{\mathrm{X}}^{\ell_{\mathrm{X}}=-1}(B)$ and $E_{\mathrm{X}}^{\ell_{\mathrm{X}}=0}(B)$ at the transition magnetic field (Supporting Information).

Although the 1D model provides an intuitive picture for the $\mathrm{X}$ in a $\mathrm{QR}$, it needs to be refined with a quasi $1 \mathrm{D}$ QR model, i.e. $2 W_{\mathrm{h}}<R_{\mathrm{e}, \mathrm{h}}$, where the finite rim widths of electrons $\left(2 W_{\mathrm{e}}\right)$ and holes $\left(2 W_{\mathrm{h}}\right)$ are considered. Provided that the radial confinement potential energy for electrons and holes can be approximated ${ }^{29,30}$ as $V_{\mathrm{e}, \mathrm{h}}(r) \sim \frac{\hbar^{2}}{2 m_{\mathrm{e}, \mathrm{h}} W_{\mathrm{e}, \mathrm{h}}^{4}}\left(r-R_{\mathrm{e}, \mathrm{h}}\right)^{2}$, the eigenenergy $\left(E_{\mathrm{e}, \mathrm{h}}^{n, m}\right)$ and eigenfunction $\left(\Psi_{\mathrm{e}, \mathrm{h}}^{n, m}\right)$ of the single-particle states can be obtained analytically for electrons and holes, which are represented by a radial quantum number $(n)$ and an angular momentum $(m) .{ }^{30}$ In order to consider the Coulomb interaction, these states were used to diagonalise the total Hamiltonian numerically. By using the exact diagonalisation method, the eigenenergies of $\mathrm{X}\left(E_{\mathrm{X}}^{\ell_{\mathrm{X}}}(B)\right)$ and $\mathrm{XX}\left(E_{\mathrm{XX}}^{\ell_{\mathrm{XX}}}(B)\right)$ can be obtained for various ring orbital angular momentum states $\left(\ell_{\mathrm{X}, \mathrm{XX}}=0, \pm 1, \pm 2, \pm 3, \cdots\right)$ (See Supporting Information).

Experimental AB oscillations of X (Fig. 4(c,d,e)) and XX (Fig. 4 (f,g,h)) were obtained after removing the fitted quadratic functions for an excitation of $P=1.2 \mathrm{kWcm}^{-2}$ (Fig. 3(a,d)), $5 P$ (Fig. 3(b,e)), and $10 P$ (Fig. 3(c,f)), respectively. The excitation dependence of $\Delta B_{\mathrm{X}}$ and $\Delta B_{\mathrm{Xx}}$ is seen clearly. For three excitations $P, 5 P$, and $10 P$, both the $\mathrm{AB}$ oscillation periods of $\mathrm{X}\left(\Delta B_{\mathrm{X}}=1.97 \mathrm{~T}, 1.85 \mathrm{~T}\right.$, and $\left.1.77 \mathrm{~T}\right)$ and $\mathrm{XX}\left(\Delta B_{\mathrm{XX}}=0.92 \mathrm{~T}, 0.68 \mathrm{~T}\right.$, and $0.48 \mathrm{~T}$ ) were observed to decrease. As the $\mathrm{AB}$ oscillations of $\mathrm{X}$ and $\mathrm{XX}$ are sensitive to the wavefunction change, $\Delta B_{\mathrm{X}}$ and $\Delta B_{\mathrm{XX}}$ can be a measure of the wavefunction susceptibility 
to excitation intensity. Although the detailed wave modification mechanism by $E_{\text {Loc }}$ has not been clarified, we suggest that the increased excitation possibly enhances $E_{\text {Loc }}$ via the trapped carriers. Suppose the trapped charge distribution is random and non-uniform, the excitation dependence can be considered in terms of $R_{\mathrm{e}, \mathrm{h}}$ and $W_{\mathrm{e}, \mathrm{h}}$ as effective parameters. Because the observed $\Delta B_{\mathrm{X}}$ and $\Delta B_{\mathrm{XX}}$ in a QR decrease with increasing excitation, it is plausible to assume large $R_{\mathrm{e}, \mathrm{h}}$ regarding $\Delta B_{\mathrm{X}}=(h / e) /\left(\pi\left|R_{\mathrm{e}}^{2}-R_{\mathrm{h}}^{2}\right|\right)$. On the other hand, the decrease of $\gamma_{\mathrm{X}}$ and $\gamma_{\mathrm{Xx}}$ with excitation (Fig. 3(h)) implies a decrease of $W_{\mathrm{e}, \mathrm{h}}$. In order to reproduce the experimental $\mathrm{AB}$ oscillation periods and diamagnetic coefficients of $\mathrm{X}$ and XX for three excitations $P, 5 P$, and $10 P$, we found the optimum parameters of $R_{\mathrm{e}}(32.0 \mathrm{~nm}$, $38.4 \mathrm{~nm}$, and $48.0, \mathrm{~nm}), R_{\mathrm{h}}(20.0 \mathrm{~nm}, 24.0 \mathrm{~nm}$, and $30.0, \mathrm{~nm})$ and $W_{\mathrm{e}, \mathrm{h}}(4.0 \mathrm{~nm}, 3.8 \mathrm{~nm}$, and $3.6 \mathrm{~nm}$ ), respectively. It is noticeable that our model does not explain how different excitation gives rise to a modification of the wavefunction in a QR. Nevertheless, we used our calculations as a reference when experiments were compared.

Given calculation results of $E_{\mathrm{X}}^{\ell_{\mathrm{X}}}(B)$ and $E_{\mathrm{XX}}^{\ell_{\mathrm{XX}}}(B)$ with the optimum parameters (Supporting Information), the AB oscillations (solid lines in Fig. 4(c,d,e,f,g,h)) were also obtained after removing the quadratic $B$-dependence terms. The experimental $\Delta B_{\mathrm{X}}$ and $\Delta B_{\mathrm{XX}}$ of both X and XX were successfully reproduced theoretically. However, a magnetic field difference $(\delta B)$ is seen as a shift in the oscillation extremum. Because the wavefunction remains localised for $B<B_{\mathrm{c}}$ due to the anisotropic $\mathrm{QR}$ structure, the $\mathrm{AB}$ oscillations begin at a finite magnetic field. It is interesting that $\delta B$ also changes for increasing excitation. This result is also consistent with our conjecture, i.e. excitation dependent $E_{\mathrm{Loc}}$ modifies the potential environment, whereby a full rotational motion around the rim becomes enabled at a different $B_{\mathrm{c}}$.

Our quasi- 1 model is also useful to estimate the degree of localisation in wavefunctions of both electron and hole. Given the optimum parameters, we calculated the probability of finding either an electron $\left(\rho_{\mathrm{ee}}\right)$ or a hole $\left(\rho_{\mathrm{he}}\right)$ at the position of a fixed electron (See Supporting Information). For example, with the optimum condition $\left(R_{\mathrm{e}}=32.0 \mathrm{~nm}, R_{\mathrm{h}}=20.0 \mathrm{~nm}\right.$, 
and $\left.W_{\mathrm{e}, \mathrm{h}}=4.0 \mathrm{~nm}\right)$, we found the $\rho_{\mathrm{he}}$ of $\mathrm{X}$ is comparable to $\xi_{\mathrm{X}} \sim 25^{\circ}$ in Fig. $4(\mathrm{a})$. This result is also in agreement with the $1 \mathrm{D}$ model, where an electron-hole pair in a $\mathrm{QR}$ can be considered as a single particle. On the other hand, in the case of XX, the four-body Coulomb interaction can be glimpsed through the distribution of the other electron $\left(\rho_{\mathrm{ee}}\right)$ and the two holes $\left(\rho_{\text {he }}\right)$ with respect to a fixed electron separately (Supporting Information), and this result is also in an agreement with our 1D model, i.e. a pair of Xs in our QR are localised at opposite positions as a consequence of the dipole-dipole interaction (Fig. 4(b)). Because the dipole-dipole interaction between the two Xs is relatively weak compared to the Coulomb between the electron and the hole, $\xi_{\mathrm{XX}} \sim 71^{\circ}$ was obtained.

As shown in Fig. 4(i), we found that the XX binding energy in the absence of $B$ decreases with increasing excitation. The decreased binding energy of XX for increasing excitation is consistent with the enlarged orbits of XX in our model. The enlarged orbit radii result in a short $\mathrm{AB}$ oscillation period. For increasing excitation from $P$ to $10 P$, the $\Delta B_{\mathrm{X}}$ and $\Delta B_{\mathrm{X}}$ becomes decreased by $10 \%$ and $40 \%$, respectively. According to the $1 \mathrm{D}$ model, the two-X WM criterion is given in the limited condition of a 1D structure, where the wavefunction localisation in a widthless rim is strong enough to assume the second order approximation for $\phi$ and $\theta$. Specifically, two Xs can be bound as a dimer as long as the pair of Xs fulfills $\xi_{\mathrm{Xx}}<\pi / 2$, whereby the total energy is dominated in the CM coordinate and the decoupled motion in the relative coordinate becomes a constant. However, when finite rim widths are considered in the quasi-1D model, the two-X WM condition looks sensitive to the wavefunction shape. Although $\Delta B_{\mathrm{X}} / \Delta B_{\mathrm{XX}}=2$ can be obtained in the $1 \mathrm{D}$ model by using Eq. (2) and Eq. (4), $\Delta B_{\mathrm{X}} / \Delta B_{\mathrm{XX}}$ becomes increased up to 3.72 with increasing excitation (Fig. 4 (j)). Therefore, the decreased XX binding energy seems to deteriorate the WM nature of the two-body rigidity, and this may explain why a subtle deviation from the XX WM condition $\left(\Delta B_{\mathrm{X}} / \Delta B_{\mathrm{XX}}>2\right)$ emerges for strong excitation.

In summary, we have found that both $\mathrm{X}$ and XX in a single GaAS/AlGaAs QR show an excitation dependence in the $\mathrm{AB}$ oscillation periods and the diamagnetic coefficients with a 
spectral blueshift. This can possibly be attributed to a modification of the wavefunctions by optically controllable $E_{\mathrm{Loc}}$ at interface defects. While the XX binding energy decreases for increased excitation, we also found that a gradual deviation from the XX-WM condition $\left(\Delta B_{\mathrm{X}} / \Delta B_{\mathrm{XX}}=2\right)$ occurs.

\section{Acknowledgement}

This work was supported by Korean Grant (NRF-2017R1A2B4011594, Pioneer Research 2013M3C1A3065522), JSPS KAKENHI Grant (JP15H05870, JP26220711) and Fundamental Research Funds for Central University of China.

\section{Supporting Information Available}

One-dimensional and quasi-one dimensional models of excitons and biexcitons in a QR were explained in detail, where the eigenenergies and the two-body probability density were calculated.

\section{References}

(1) H. Bary-Soroker et al. Phys. Rev. Lett. 101, 057001 (2008).

(2) A. J. M. Giesbers et al. Nat. Phys. 6, 173 (2010).

(3) A. van Oudenaarden et al. Nature 391, 768 (1998).

(4) A. Lorke et al. Phys. Rev. Lett. 84, 2223 (2000).

(5) N. A. J. M. Kleemans et al. Phys. Rev. Lett. 99, 146808 (2007).

(6) A. O. Govorov et al. Phys. Rev. B 66, 081309(R) (2002).

(7) I. L. Kuskovsky et al. Phys. Rev. B 76, 035342 (2007). 
(8) I. R. Sellers et al. Phys. Rev. Lett. 100, 136405 (2008).

(9) H. D. Kim et al. Nano Lett. 16, 27 (2016).

(10) S. Miyamoto et al. Phys. Rev. B 82, 073306 (2010).

(11) E. Ribeiro et al. Phys. Rev. Lett. 92, 126402 (2004).

(12) M. D. Teodoro et al. Phys. Rev. Lett. 104, 086401 (2010).

(13) S. Pecker et al. Nat. Phys. 9, 576 (2013).

(14) C. Ellenberger et al. Phys. Rev. Lett. 96, 126806 (2006).

(15) A. Singha et al. Phys. Rev. Lett. 104, 246802 (2010).

(16) R. Egger et al. Phys. Rev. Lett. 82, 3320 (1999).

(17) R. Okuyama et al. Phys. Rev. B 83, 195311 (2011).

(18) K. Niemela et al. Europhys. Lett. 36, 533 (1996).

(19) T. Chakraborty et al. Phys. Rev. B 50, 8460 (1994).

(20) T. Chwiej et al. Phys. Rev. B 79, 085305 (2009).

(21) S. Sanguinetti et al. J. Crys. Growth 253, 71 (2003).

(22) T. Mano et al. J. Crys. Growth 301, 740 (2007).

(23) H. D. Kim et al. Appl. Phys. Lett. 102, 033112 (2013).

(24) T. C. Lin et al. Phys. Rev. B 80, 081304(R) (2009).

(25) H. D. Kim et al. Sci. Rep. 7, 40026 (2017).

(26) H. D. Kim et al. Appl. Phys. Lett. 103, 173106 (2013).

(27) J. A. Barker et al. Phys. Rev. B 69, 035327 (2004). 
(28) V. V. Arsoski et al. Phys. Rev. B 87, 085314 (2013).

(29) C. González-Santander et al. Phys. Rev. B 84, 235103 (2011).

(30) W. C. Tan et al. Seimicond. Sci. Technol. 11, 1635 (1996). 
Graphical TOC Entry

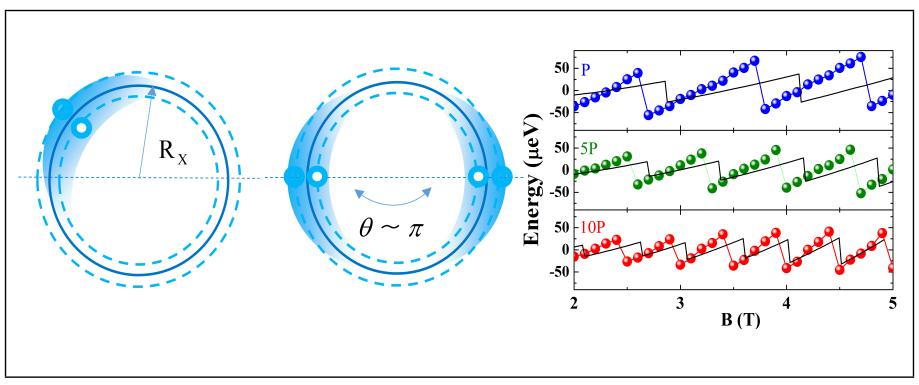

